Pacific Journal of Mathematics

INVERSE SPECTRAL PROBLEMS FOR CERTAIN 


\title{
INVERSE SPECTRAL PROBLEMS FOR CERTAIN DIFFERENTIAL OPERATORS
}

\author{
RODERIC MURUfaS
}

Let $L_{0}$ be a given differential operator with spectral matrix $\left(\rho_{i j}^{0}\right)$. There is a concept of "closeness to $\left(\rho_{i j}^{0}\right)$ " such that for every positive matrix measure $\left(\rho_{i j}\right)$ which is "close to $\left(\rho_{i j}^{0}\right)$ " there exists some differential operator $L$ for which $\left(\rho_{i j}\right)$ is a spectral matrix and there exists a potentially computational technique by which $L$ may be constructed from $\left(\rho_{i j}\right)$ and $\left(\rho_{i j}^{0}\right)$. The formulation of the "closeness to $\left(\rho_{i j}^{0}\right)$ " concept and the presentation of the techniques by which $L$ may be constructed from $\left(\rho_{i j}\right)$ and $\left(\rho_{i j}^{0}\right)$ are referred to as the local inverse spectral problem, which is the subject of this paper.

Introduction. Sahnovič [6] has presented a formulation of the local inverse spectral problem but he defines "closeness to $\left(\rho_{i j}^{0}\right)$ " in a manner that is too restrictive and excludes many solvable cases. For example many problems in the second order case, which had previously been solved by Gelfand and Levitan [2], do not meet the "closeness" criterion of Sahnovič. On the other hand his presentation omits some necessary technical conditions that, despite their awkward appearance, must be assumed in case $2 n>2$.

The present article gives the least restrictive conditions possible, which in the second order case coincide with the conditions given by Gelfand and Levitan.

The above changes require modifications of the technique by which the differential operator $L$ is constructed from $\left(\rho_{i j}\right)$ and $\left(\rho_{i j}^{0}\right)$.

\section{CHAPTER 1 \\ TeChNical Preliminaries}

1. Orientation. Let $l$ be the differential expression defined by

$$
l u=(-1)^{n} u^{(2 n)}+(-1)^{n-1}\left(p_{1} u^{(n-1)}\right)^{(n-1)}+\cdots+p_{n} u,
$$

where the coefficients $p_{k}(x)$ are real valued functions on $[0, \infty)$ that are locally integrable. Any formally selfadjoint differential expression defined on the positive real axis that is regular at zero and has sufficiently differentiable real coefficients can be put into this form if $p_{0} \equiv 1$. On the 
other hand, assuming the given form initially allows weakening the differentiability requirements on the coefficients. This is done by defining quasi-derivatives with respect to $l$ :

$$
\begin{aligned}
u^{[k]} & =u^{(k)}, \quad k=0,1, \ldots, n, \\
u^{[n+k]} & =\sum_{i=0}^{k}(-1)^{l}\left(p_{k-\imath} u^{(n-k+i)}\right)^{(i)}, \quad k=1,2, \ldots, n,
\end{aligned}
$$

where $p_{0} \equiv 1$. Now $l u=u^{[2 n]}$ can be defined for all functions $u$ such that $u^{[k]}$ is absolutely continuous for $k=0,1, \ldots, 2 n-1$. These quasi-derivatives enable certain formal simplifications and are therefore convenient even when the usual smoothness is present or required.

An $n \times 2 n$ constant matrix $A=\left(a_{l j}\right)$ is said to represent selfadjoint boundary conditions if $A$ is real, has rank $n$ and satisfies

$$
\sum_{k=1}^{n} a_{i k} a_{J, 2 n+1-k}=\sum_{k=1}^{n} a_{l, n+k} a_{j, n+1-k} \quad \text { for } i, j=1, \ldots, n .
$$

Now $n$ boundary conditions are specified by the equation $A \cdot \hat{u}(0)=(0)$, where $\hat{u}(0)$ denotes the column vector

$$
\left(u(0), u^{[1]}(0), \ldots, u^{[2 n-1]}(0)\right)^{\mathrm{T}} .
$$

It is easily seen that for any real, invertible $n \times n$ matrix $N$, the matrix $N \cdot A$ still represents selfadjoint boundary conditions. Furthermore, they are equivalent to $A$ in terms of the effect on the domain of functions satisfying these boundary conditions.

Given a formally selfadjoint differential expression

$$
l u=(-1)^{n} u^{(2 n)}+(-1)^{n-1}\left(p_{1}(x) u^{(n-1)}\right)^{(n-1)}+\cdots+p_{n}(x) u
$$

with real, locally integrable coefficients $p_{\imath}$ for $i=1,2, \ldots, n$ defined on the interval $[0, \infty)$ and a set of selfadjoint boundary conditions $A$, we will define a symmetric differential operator $L=L(l, A)$ using quasi-derivatives as follows.

Let

$$
\begin{aligned}
& \mathscr{D}^{\prime}=\left\{f(x) \in \mathcal{L}^{2}[0, \infty) \mid f^{[k]} \in A, C,[0, \infty)\right. \\
& \left.\quad \text { for } k=0,1, \ldots, 2 n-1 ; f^{[2 n]} \in \mathcal{L}^{2}[0, \infty)\right\} .
\end{aligned}
$$

This is the largest set of functions on which $l$ may be defined and still determine an operator on $\mathfrak{L}^{2}[0, \infty)$. Corresponding to $l$ define a bilinear form $\langle\cdot, \cdot\rangle$ for functions $\xi, \eta \in \mathbb{D}^{\prime}$ by

$$
\langle\xi, \eta\rangle=\sum_{k=1}^{n}\left(\xi^{[k-1]} \bar{\eta}^{[2 n-k]}-\xi^{[2 n-k]} \bar{\eta}^{[k-1]}\right) .
$$


When $\xi, \eta$ and the coefficients are sufficiently differentiable,

$$
\begin{aligned}
\langle\xi, \eta\rangle=\sum_{m=1}^{n}(-1)^{m}\left[\sum_{i=0}^{m-1}(-1)^{i} \bar{\eta}^{(l)}\left(p_{n-m} \xi^{(m)}\right)^{(m-1-i)}\right. & \\
& \left.\quad-\sum_{i=0}^{m-1}(-1)^{i} \xi^{(i)}\left(p_{n-m} \bar{\eta}^{(m)}\right)^{(m-1-l)}\right] .
\end{aligned}
$$

Now for $\xi, \eta \in \mathscr{Q}^{\prime}$ we can write

$$
\int_{0}^{x}[l \xi] \bar{\eta}-\int_{0}^{x} \xi[\overline{l \eta}]=\langle\xi(x), \eta(x)\rangle-\langle\xi(0), \eta(0)\rangle .
$$

This is the integral form of Lagrange's identity. It may be noted that $A \cdot \hat{\xi}(0)=A \cdot \hat{\eta}(0)=(0)$ implies $\langle\xi(0), \eta(0)\rangle=0$ whenever $A$ represents selfadjosint boundary conditions.

Now let

$$
\mathscr{D}=\left\{f(x) \in \mathscr{D}^{\prime} \mid A \hat{f}(0)=(0) \quad \text { and } \quad \lim _{x \rightarrow \infty}\langle f(x), g(x)\rangle=0 \forall g \in \mathscr{D}^{\prime}\right\}
$$

and define $L u=l u$ for $u \in \mathscr{D}$. The adjoint operator to $L$ has domain

$$
\mathscr{D}^{*}=\left\{f(x) \in \mathscr{D}^{\prime} \mid A \hat{f}(0)=(0)\right\}
$$

so $L$ is symmetric and in some cases selfadjoint.

Let the components of the row vector $u(x, \lambda)=\left(u_{1}(x, \lambda), \ldots\right.$, $\left.u_{n}(x, \lambda)\right)$ be a set of $n$ linearly independent solutions to the boundary value problem $l w=\lambda w, A \cdot \hat{w}(0, \lambda)=(0)$ which satisfy the condition that the $2 n \times n$ matrix $\hat{u}(0, \lambda)$ be independent of $\lambda$. Such a vector is said to represent generalized eigenfunctions for $L$ since the components need not be in $\mathscr{D}$. The condition that $\hat{u}(0, \lambda)$ be independent of $\lambda$ is a normalization which simplifies matters because it implies that any two generalized eigenfunctions $u$ and $\tilde{u}$ for $L$ are related by the equation $u(x, \lambda)=\tilde{u}(x, \lambda)$ - $\delta$, where $\delta$ is some $n \times n$ invertible constant matrix.

For example, in the second order case if $l w=-w^{\prime \prime}$ and $A=\left(\begin{array}{ll}0 & 1\end{array}\right)$, then for any function $f(\lambda)$ we can see that $u(x, \lambda)=f(\lambda) \cdot \cos (\sqrt{\lambda} x)$ is a solution to the boundary value problem $l w=\lambda w, A \cdot \hat{w}(0, \lambda)=(0)$ since

$$
\left(\begin{array}{ll}
0 & 1
\end{array}\right)\left(\begin{array}{c}
f(\lambda) \cos (0) \\
-\sqrt{\lambda} f(\lambda) \sin (0)
\end{array}\right)=0 .
$$

However, requiring

$$
\hat{u}(0, \lambda)=\left(\begin{array}{c}
f(\lambda) \\
0
\end{array}\right)
$$


to be independent of $\lambda$ allows only constant multiples of $\cos (\sqrt{\lambda} x)$.

Similarly in the case

$$
l w=w^{\prime \prime \prime \prime}, \quad A=\left(\begin{array}{cccc}
0 & 1 & 0 & 0 \\
0 & 0 & 0 & 1
\end{array}\right)
$$

we restrict ourselves to independent linear combinations of

and

$$
u_{1}(x, \lambda)=\frac{\cosh \left(\lambda^{1 / 4} x\right)+\cos \left(\lambda^{1 / 4} x\right)}{2}
$$

$$
u_{2}(x, \lambda)=\frac{\cosh \left(\lambda^{1 / 4} x\right)-\cos \left(\lambda^{1 / 4} x\right)}{2 \lambda^{2 / 4}} .
$$

Now given the symmetric differential operator $L$ and a vector $u(x, \lambda)$ of generalized eigenfunctions, let $\tilde{L}$ be any fixed selfadjoint extension of $L$. It is well known [4] that there exists a unique positive matrix measure $\left(\rho_{i j}\right)$, herein referred to as the spectral matrix for $\tilde{L}$ corresponding to $u(x, \lambda)$, for which the map $U$ defined by

$$
U[f(x)]=F(\lambda)=\int_{0}^{\infty} u(x, \lambda) f(x) d x
$$

is an isometry of $\mathcal{L}^{2}[0, \infty)$ onto $\mathcal{L}^{2}\left(\mathbf{R},\left(\rho_{i j}\right)\right)$ such that

$$
\begin{gathered}
U\left(\mathscr{D}_{\tilde{L}}\right)=\mathscr{\mathscr { D }}_{\Lambda}=\left\{F(\lambda) \in \mathcal{L}^{2}\left(\mathbf{R},\left(\rho_{i j}\right)\right) \mid \lambda F(\lambda) \in \mathcal{L}^{2}\left(\mathbf{R},\left(\rho_{i j}\right)\right)\right\}, \\
U^{-1}[F(\lambda)]=f(x)=\int_{\mathbf{R}} F(\lambda) d\left(\rho_{i j}\right) u^{*}(x, \lambda),
\end{gathered}
$$

and for $f \in \mathscr{D}_{\tilde{L}}$ we have $\tilde{L} f=U^{-1} \Lambda U[f]$. Furthermore if $f, g \in \mathcal{L}^{2}[0, \infty)$, $F(\lambda)=U[f]$ and $G(\lambda)=U[g]$, then

$$
\int_{0}^{\infty} f(x) \bar{g}(x) d x=\int_{\mathbf{R}} F(\lambda) d\left(\rho_{i j}(\lambda)\right) G^{*}(\lambda) .
$$

This last equation is the generalized Parseval equality.

The relations between $\tilde{L}$ and $\left(\rho_{i j}\right)$ induce similar ones between $L$ and $\left(\rho_{i j}\right)$, some of which allow $\left(\rho_{i j}\right)$ to be used to determine $L$ in a manner which is the subject of this article. Since the surjectivity of the map $U$ is not essential we give the following definition.

2. Definition. Let $L(l, A)$ be a symmetric differential operator of order $2 n$ on the interval $[0, \infty)$ and let $u(x, \lambda)$ be a $1 \times n$ vector of generalized eigenfunctions of $L$. A positive matrix measure $\left(\rho_{i j}\right)$ will be called a determining matrix for $L$ corresponding to $u(x, \lambda)$ if the map $U$ defined by

$$
U[f(x)]=\int_{0}^{\infty} f(x) u(x, \lambda) d x
$$


gives an isometry of $\mathcal{L}^{2}[0, \infty)$ into $\mathcal{L}^{2}\left(\mathbf{R},\left(\rho_{i j}\right)\right)$ such that

$$
U\left(\mathscr{D}_{L}\right) \subset \mathscr{D}_{\Lambda}=\left\{F(\lambda) \in \mathcal{L}^{2}\left(\mathbf{R},\left(\rho_{i j}\right)\right) \mid \lambda F(\lambda) \in \mathcal{L}^{2}\left(\mathbf{R},\left(\rho_{i j}\right)\right)\right\} .
$$

For $f(x) \in \mathscr{Q}_{L}$ we have $U \circ L[f]=\Lambda \circ U[f]$, and for $f, g \in \mathcal{L}^{2}[0, \infty)$ we have

$$
\int_{0}^{\infty} f(x) \bar{g}(x) d x=\int_{\mathbf{R}} F(\lambda) d\left(\rho_{i j}(\lambda)\right) G^{*}(\lambda)
$$

where $F(\lambda)=U[f]$ and $G(\lambda)=U[g]$.

3. REMARK. The spectral matrix for any selfadjoint extension of $L$ corresponding to $u(x, \lambda)$ is a determining matrix for $L$ corresponding to $u(x, \lambda)$. In particular, when $L$ is selfadjoint then the unique spectral matrix for $L$ corresponding to $u(x, \lambda)$ is a determining matrix for $L$ corresponding to $u(x, \lambda)$.

An example of a determining matrix that is not a spectral matrix is given in the appendix for a nonselfadjoint Sturm-Liouville operator. This example, by the way, illustrates the need for some clarification in the articles of Gelfand and Levitan [2] and of Sahnovič [6].

4. Notation. Throughout the remaining chapters the following notations will be used.

Let

$$
\begin{aligned}
l^{1} u & =(-1)^{n} u^{(2 n)}+(-1)^{n-1}\left(p_{1}(x) u^{(n-1)}\right)^{(n-1)}+\cdots+p_{n}(x) u \\
& =(-1)^{n} u^{(2 n)}+\tilde{p}_{2 n-2}(x) u^{(2 n-2)}+\cdots+\tilde{p}_{0} u
\end{aligned}
$$

define a formally selfadjoint differential expression with real coefficients $p_{i} \in C_{[0, \infty)}^{n-i}$ for $i=1, \ldots, n$ and let $A$ represent a set of $n$ selfadjoint boundary conditions at zero. Let $\left\{u_{i}(x, \lambda)\right\}_{i=1}^{n}$ be some set of linearly independent generalized eigenfunctions for $L^{1}=L\left(l^{1}, A\right)$. Let $\left(\rho_{i j}^{1}\right)$ be a determining matrix for $L^{1}$ corresponding to $\left(u(x, \lambda), \ldots, u_{n}(x, \lambda)\right)$ and let $\varphi(x, \lambda)=\int_{0}^{x} u(y, \lambda) d y$.

Let

$$
l^{2} v=(-1)^{n} v^{(2 n)}+\tilde{q}_{2 n-2}(x) v^{(2 n-2)}+\cdots+\tilde{q}_{0}(x) v
$$

define another differential expression with continuous coefficients on $[0, \infty)$ and let $B$ be some set of $n$ linearly independent boundary conditions at zero. In case $l^{2}$ and $B$ are formally selfadjoint they define a 
symmetric differential operator $L^{2}=L\left(l^{2}, B\right)$, where $l^{2}$ may now be put into the form

$$
l^{2} v=(-1)^{n} v^{(2 n)}+(-1)^{n-1}\left(q_{1}(x) v^{(n-1)}\right)^{(n-1)}+\cdots+q_{n}(x) v .
$$

In this case let $\left\{v_{i}(x, \lambda)\right\}_{i=1}^{n}$ be a set of linearly independent generalized eigenfunctions for $L^{2}$, let $\left(\rho_{i j}^{2}\right)$ be a determining matrix for $L^{2}$ corresponding to $v(x, \lambda)=\left(v_{1}(x, \lambda), \ldots, v_{n}(x, \lambda)\right)$, and let $\left(\sigma_{i j}\right)=\left(\rho_{i j}^{2}-\rho_{i j}^{1}\right)$. The hermitian matrix measure $\left(\sigma_{i j}\right)$ is in general not positive semi-definite. Nevertheless, certain integrals are formally definable. In particular for the vector valued functions

$$
f(\lambda)=\left(f_{1}(\lambda), \ldots, f_{n}(\lambda)\right) \text { and } g(\lambda)=\left(g_{1}(\lambda), \ldots, g_{n}(\lambda)\right),
$$

the notation $\int f(\lambda) d\left(\sigma_{i j}(\lambda)\right) g^{*}(\lambda)$ will be used to denote the integral

$$
\int \sum_{i, j} f_{i}(\lambda) \bar{g}_{j}(\lambda) d \sigma_{i j}(\lambda)=\int f(\lambda)\left(n_{i j}(\lambda)\right) g^{*}(\lambda) d \sigma(\lambda),
$$

where $\sigma$ is a one-dimensional measure with respect to which each entry of $\left(\sigma_{i j}\right)$ is absolutely continuous and where $\left(n_{i j}(\lambda)\right)=\left(d \sigma_{i j} / d \sigma\right)$.

Now having defined $L^{1}=L\left(l^{1}, A\right)$ and $L^{2}=L\left(l^{2}, B\right)$ as outlined, let $p_{0}=q_{0} \equiv 1$ and let $\langle\cdot, \cdot\rangle_{1}$ and $\langle\cdot, \cdot\rangle_{2}$ be the bilinear forms corresponding to $l^{1}$ and $l^{2}$ respectively. Also let $\tilde{q}_{2 n}=\tilde{p}_{2 n} \equiv(-1)^{n}$ and $\tilde{q}_{2 n-1}=$ $\tilde{p}_{2 n-1} \equiv 0$.

When $l^{1}, l^{2}$ and a function $K(x, y) \in C^{2 n}(0 \leq y \leq x)$ are given, then for later expository clarity let $\alpha_{r}(x)$ represent the expression defined by

$$
\begin{aligned}
\alpha_{r}(x)= & \sum_{i=[(r+1) / 2]}^{\min (r, n-1)}(-1)^{i}\left(\begin{array}{c}
i \\
r-i
\end{array}\right)\left[q_{n-i}^{(2 i-r)}-p_{n-i}^{(2 i-r)}\right] \\
& +\sum_{m=r+1}^{2 n} \sum_{k=[(m+1) / 2]}^{\min (m, n-1)}(-1)^{k}\left(\begin{array}{c}
k \\
m-k
\end{array}\right) q_{n-k}^{(2 k-m)} \\
& \times \sum_{i=0}^{m-r-1}\left(\begin{array}{c}
i+r \\
r
\end{array}\right) K_{m-r-i-1,0}^{(i)}(x, x) \\
& +\sum_{m=r+1}^{n}(-1)^{m+r} \frac{\partial^{m-1-r}}{\partial t^{m-1-r}}\left[p_{n-m}^{(t)} K_{0, m}^{(x, t)}\right]_{t=x} \\
& -\sum_{m=1}^{\min (r, n)}(-1)^{m} \sum_{i=0}^{2 m-1-r}(-1)^{i}\left(\begin{array}{c}
m-1-i \\
r-m
\end{array}\right) \\
& \times P_{n-m}^{(2 m-1-i-r)}(x) K_{0, i}(x, x)
\end{aligned}
$$

for $r=0,1, \ldots, 2 n-2$. 
An expression like $K_{a, b}^{(c)}(x, x)$ symbolizes taking $a$ partial derivatives of $K(x, y)$ with respect to the first variable, $b$ partial derivatives with respect to the second variable, evaluating the result on the diagonal $y=x$, and then taking $c$ more derivatives of the resulting function of $x$.

5. REMARKs. Two explanatory remarks are in order here concerning the usage of the expression $\alpha_{r}(x)$.

First, given two symmetric differential operators $L^{1}$ and $L^{2}$ and a function $K(x, y)$, then the consistency of the $2 n-1$ conditions $\alpha_{r}(x)=0$, $r=0,1, \ldots, 2 n-2$, is dependent on $K$. These conditions impose $2 n-1$ relations on $K$ and several of its derivatives on the diagonal $y=x$.

On the other hand suppose a symmetric differential operator $L^{1}$ and a function $K(x, y)$ are given. If $r$ is even, $\alpha_{r}(x)$ involves only those functions $q_{i}$ where $i \leq n-r / 2$, so starting with the condition $\alpha_{2 n-2}(x)=$ 0 and using conditions with successively smaller even indices, one may successively define the real functions $q_{1}, q_{2}, \ldots, q_{n}$. Here the consistency of the remaining $n-1$ conditions $\alpha_{r}(x)=0, r=1,3,5, \ldots, 2 n-3$, is dependent on $K$.

6. Definition. Given a symmetric differential operator $L^{1}$ and a $1 \times n$ row vector $u(x, \lambda)$ representing a choice of generalized eigenfunctions for $L^{1}$, an $n \times n$ positive matrix measure $\left(\rho_{i j}\right)$ will be called adequate with respect to $u(x, \lambda)$ if whenever $g \in \mathcal{L}^{2}[0, \infty)$ has compact support and we put $G(\lambda)=\int_{0}^{\infty} g(x) u(x, \lambda) d x$, then $\int_{\mathbf{R}} G(\lambda) d\left(\rho_{i j}\right) G^{*}(\lambda)=0$ implies $g \equiv 0$.

7. Remark. Parseval's equality insures that any determining matrix ( $\left.\rho_{i j}^{1}\right)$ for $L^{1}$ corresponding to $\left\{u_{i}(x, \lambda)\right\}_{i=1}^{n}$ is adequate with respect to $u(x, \lambda)$ since

$$
\int_{\mathbf{R}} G(\lambda) d\left(\rho_{i j}^{1}\right) G^{*}(\lambda)=\int_{0}^{\infty} g^{2}(x) d x
$$

8. Definition. Given some kernel $K(x, y)$ let $\mathscr{K}$ denote the operator

$$
\mathcal{K}[f]=f(x)+\int_{0}^{x} K(x, y) f(y) d y .
$$

9. Definition. Given the symmetric differential operators $L^{1}$ and $L^{2}$, the expression $\mathscr{K} L^{1}=L^{2} \mathcal{K}$ will mean there exists a real kernel $K(x, y) \in$ $C^{2 n}$ for $0 \leq y \leq x$ such that $v(x, \lambda)=\mathscr{K}[u(x, \lambda)]$ is a generalized eigenfunction for $L^{2}$ whenever $u(x, \lambda)$ is a generalized eigenfunction for $L^{1}$. 
10. REMARK. If $\mathscr{K} L^{1}=L^{2} \mathcal{K}$ and $\left\{u_{i}(x, \lambda)\right\}_{i=1}^{n}$ is a linearly independent set of generalized eigenfunctions for $L^{1}$, then $\left\{\mathscr{K}\left[u_{i}(x, \lambda)\right]\right\}_{l=1}^{n}$ is a linearly independent set of generalized eigenfunctions for $L^{2}$. This is because the equation $u(x)=-\int_{0}^{x} K(x, y) u(y) d y$ has only the trivial solution, as can be seen by successive estimates. Therefore $\mathcal{K}$ is injective.

11. Lemma. Given two symmetric differential operators $L^{1}$ and $L^{2}$ such that $\mathscr{K} L^{1}=L^{2} \mathcal{K}$, let the vector $u(x, \lambda)$ represent $n$ linearly independent generalized eigenfunctions for $L^{1}$ and let $v(x, \lambda)=\mathscr{K}[u(x, \lambda)]$. Let $\left(\rho_{i j}^{1}\right)$ be a determining matrix for $L^{1}$ corresponding to $u(x, \lambda)$ and let $\left(\rho_{i j}^{2}\right)$ be a determining matrix for $L^{2}$ corresponding to $v(x, \lambda)$. Let $\left(\sigma_{i j}\right)=\left(\rho_{i j}^{2}-\rho_{i j}^{1}\right)$, $\mathcal{H}$ the operator inverse to $\mathcal{K}, H(x, y)$ its kernel and $\varphi(x, \lambda)=\int_{0}^{x} u(t, \lambda) d t$. Then

$$
f(x, y)=\int_{\mathbf{R}} \varphi(x, \lambda) d\left(\sigma_{\imath j}(\lambda)\right) \varphi^{*}(y, \lambda)
$$

exists and has a mixed derivative $F(x, y)=\partial^{2} f / \partial x \partial y$ which is uniquely determined by $K(x, y)$ such that

$$
F(x, y)=H(x, y)+\int_{0}^{y} H(x, s) H(y, s) d s
$$

for $0 \leq y \leq x$. Moreover, $F(x, y) \in C^{2 n}$ for $0 \leq x, y<\infty$,

$$
F(x, y)+K(x, y)+\int_{0}^{x} K(x, s) F(s, y) d s=0
$$

for $0 \leq y \leq x$, and $\left(\rho_{l j}^{2}\right)$ is adequate with respect to $u(x, \lambda)$.

Proof. Suppose $f(x) \in C[0, \infty)$ and

$$
0=f(x)+\int_{0}^{x} K(x, y) f(y) d y
$$

that is,

$$
f(x)=-\int_{0}^{x} K(x, y) f(y) d y .
$$

This homogeneous Volterra equation has only the zero solution, as successive estimates will show. Hence $\mathcal{K}$ has a unique inverse $\mathcal{H}$ defined on the image of $\mathcal{K}$. To find an explicit representation we arbitrarily fix $i$ and consider the Volterra integral equation

$$
u_{i}(x, \lambda)=v_{i}(x, \lambda)-\int_{0}^{x} K(x, y) u_{i}(y, \lambda) d y,
$$

taking $u_{i}$ to be the unknown function. The technique of successive 
substitutions will produce a unique solution of the form

$$
u_{i}(x, \lambda)=v_{i}(x, \lambda)+\int_{0}^{x} H_{i}(x, y) v_{i}(y, \lambda) d y,
$$

where $H_{i}(x, y)=\sum_{j=1}^{\infty} K_{j}(x, y)$ and $K_{j}(x, y)$ is the so-called $j$ th iterated kernel. For example here

$$
\begin{aligned}
K_{1}(x, y) & =-K(x, y) \\
K_{2}(x, y)= & \int_{y}^{x} K\left(x, x_{1}\right) K\left(x_{1}, y\right) d x_{1}, \\
K_{3}(x, y)= & -\int_{y}^{x} \int_{y}^{x_{1}} K\left(x, x_{1}\right) K\left(x_{1}, x_{2}\right) K\left(x_{2}, y\right) d x_{2} d x_{1}, \\
& \vdots \\
K_{n+1}(x, y)= & (-1)^{n+1} \int_{y}^{x} \int_{y}^{x_{1}} \cdots \int_{y}^{x_{n-1}} K\left(x, x_{1}\right) K\left(x_{1}, x_{2}\right) \\
\cdots & \cdots\left(x_{n}, y\right) d x_{n} d x_{n-1} \cdots d x_{1} .
\end{aligned}
$$

Dropping the subscript on $H_{i}(x, y)$ because of this independence of $i$, we summarize:

$$
u_{i}(x, \lambda)=v_{i}(x, \lambda)+\int_{0}^{x} H(x, y) v_{i}(y, \lambda) d y=\mathcal{H}\left(v_{i}\right)
$$

for $i=1,2, \ldots, n$. Note also that the continuity of $H$ is easily established by estimates using the iterated kernels and the differentiability of $K$.

Now integrating

$$
u_{i}(x, \lambda)=v_{i}(x, \lambda)+\int_{0}^{x} H(x, y) v_{i}(y, \lambda) d y
$$

from 0 to $x$ we get

$$
\varphi_{i}(x, \lambda)=\int_{0}^{x}\left(1+\int_{t}^{x} H(\xi, t) d \xi\right) v_{i}(t, \lambda) d t .
$$

Define

$$
h_{x}(t)= \begin{cases}1+\int_{t}^{x} H(\xi, t) d \xi, & t \leq x, \\ 0, & t>x .\end{cases}
$$

The generalized Parseval equality implies

$$
\int_{\mathbf{R}} \varphi(x, \lambda) d\left(\rho_{i j}^{2}\right) \varphi^{*}(y, \lambda)=\int_{0}^{\infty} h_{x}(t) h_{y}(t) d t
$$


and if $y \leq x$, the right-hand side becomes

$$
\begin{aligned}
\int_{0}^{y} d t+ & \int_{0}^{y} \int_{t}^{y} H(\eta, t) d \eta d t+\int_{0}^{y} \int_{t}^{x} H(\xi, t) d \xi d t \\
& +\int_{0}^{y}\left(\int_{t}^{x} H(\xi, t) d \xi\right)\left(\int_{t}^{y} H(\eta, t) d \eta\right) d t
\end{aligned}
$$

On the other hand, since

$$
\varphi_{i}(x, \lambda)=\int_{0}^{\infty} \chi_{[0, x]}(\zeta) u_{i}(\zeta, \lambda) d \zeta,
$$

by Parseval's equality,

$$
\int_{0}^{y} d t=\int_{\mathbf{R}} \varphi(x, \lambda) d\left(\rho_{i j}^{1}\right) \varphi^{*}(y, \lambda),
$$

and subtracting this from the above equation gives

$$
\begin{aligned}
f(x, y) \triangleq & \int_{\mathbf{R}} \varphi(x, \lambda) d\left(\sigma_{\imath j}\right) \varphi^{*}(y, \lambda) \\
= & \int_{0}^{y} \int_{t}^{y} H(\eta, t) d \eta d t+\int_{0}^{y} \int_{t}^{x} H(\xi, t) d \xi d t \\
& +\int_{0}^{y}\left(\int_{t}^{x} H(\xi, t) d \xi\right)\left(\int_{t}^{y} H(\eta, t) d \eta\right) d t .
\end{aligned}
$$

Now, since the right-hand side exists and has a mixed derivative, the left-hand side does too, and in fact

$$
F(x, y) \triangleq \frac{\Delta}{=} \frac{\partial^{2} f}{\partial x \partial y}=H(x, y)+\int_{0}^{y} H(x, t) H(y, t) d t \quad \text { for } y \leq x .
$$

This representation shows the continuity of $F$ and its independence from the choices of $\left(\rho_{i j}^{1}\right)$ and $\left(\rho_{i j}^{2}\right)$. In fact $H$ and $F$ are uniquely determined by $K(x, y)$.

The next step is to develop the integral equation in volving $K$ and $F$.

Suppose $0 \leq b \leq y \leq a \leq x$. We have

$$
\begin{aligned}
\int_{b}^{y} u_{j}(t, \lambda) d t= & \int_{b}^{y}\left[v_{j}(t, \lambda)+\int_{0}^{t} H(t, s) v_{j}(s, \lambda) d s\right] d t \\
= & \int_{b}^{y} v_{j}(s, \lambda) d s+\int_{0}^{b} v_{j}(s, \lambda)\left(\int_{b}^{y} H(t, s) d t\right) d s \\
& +\int_{b}^{y} v_{j}(s, \lambda)\left(\int_{s}^{y} H(t, s) d t\right) d s \\
= & \int_{0}^{\infty} v_{j}(s, \lambda) g_{b y}(s) d s,
\end{aligned}
$$


where we let

$$
g_{b y}(s)=\chi_{[b, y]}(s)\left[1+\int_{s}^{y} H(t, s) d t\right]+\chi_{[0, b)}(s) \cdot \int_{b}^{y} H(t, s) d t .
$$

Now using (1), Parseval's equality and the fact that $a \geq y$, the integral

$$
q \triangleq \int_{-\infty}^{\infty} \sum_{i, j}\left(\int_{a}^{x} v_{i}(t, \lambda) d t\right)\left(\int_{b}^{y} u_{j}(t, \lambda) d t\right) d \rho_{i j}^{2}(\lambda)
$$

may be written

$$
\begin{aligned}
g= & \int_{-\infty}^{\infty} \sum_{i, j}\left(\int_{0}^{\infty} v_{t}(t, \lambda) \chi_{[a, x]}(t) d t\right) \\
& \times\left(\int_{0}^{\infty} v_{j}(t, \lambda) g_{b y}(t) d t\right) d \rho_{i j}^{2}(\lambda) \\
= & \int_{0}^{\infty} \chi_{[a, x]}(t) g_{b y}(t) d t=\int_{a}^{x} g_{b y}(t) d t=0 .
\end{aligned}
$$

Now in writing 9 we use the identity

$$
\begin{aligned}
\int_{a}^{x} v_{l}(t, \lambda) d t= & \int_{a}^{x}\left(u_{i}(t, \lambda)+\int_{0}^{t} K(t, r k) u_{i}(r, \lambda) d r\right) d t \\
= & \int_{a}^{x} u_{l}(t, \lambda) d t+\int_{0}^{a} u_{l}(r, \lambda) \int_{a}^{x} K(t, r) d t d r \\
& +\int_{a}^{x} u_{l}(r, \lambda) \int_{r}^{x} K(t, r) d t d r
\end{aligned}
$$

and subtract

$$
\begin{gathered}
\int_{-\infty}^{\infty} \sum\left(\int_{a}^{x} u_{i}(s, \lambda) d s\right)\left(\int_{b}^{y} u_{J}(s, \lambda) d s\right) d \rho_{i j}^{1}(\lambda) \\
=\int_{0}^{\infty} \chi_{[a, x]}(s) \cdot \chi_{[b, y]}(s) d s=0 .
\end{gathered}
$$

Rewriting and using Parseval's equality it can be shown that

$$
\begin{gathered}
f(x, y)-f(x, b)-f(a, y)+f(a, b) \\
\quad+\int_{a}^{x} \int_{0}^{t} K(t, s)\left[f_{s}(s, y)-f_{s}(s, b)\right] d s d t \\
+\int_{b}^{y} \int_{a}^{x} K(t, s) d t d s=0 .
\end{gathered}
$$

Finally, differentiating with respect to $x$ and $y$ we get

$$
F(x, y)+\int_{0}^{x} K(x, s) F(s, y) d s+K(x, y)=0 \text { for } 0 \leq y \leq x .
$$


Since $F$ is continuous and $K \in C^{2 n}$ for $0 \leq y \leq x$, then from this integral equation it is easily seen that as a function of $x, F$ is as smooth as $K$. Further, the symmetry of $F, F(x, y)=F(y, x)$, implies $F(x, y) \in C^{2 n}$ for $0 \leq x, y<\infty$.

Finally we must show $\left(\rho_{i j}^{2}\right)$ is adequate with respect to $u(x, \lambda)$.

Let $g(x)$ be an element of $\varrho^{2}[0, \infty)$ with compact support.

$$
\begin{aligned}
G_{i}(\lambda) & \stackrel{\Delta}{=} \int_{0}^{\infty} g(x) u_{i}(x, \lambda) d x \\
& =\int_{0}^{\infty} g(x)\left(v_{i}(x, \lambda)+\int_{0}^{x} H(x, t) v_{i}(t, \lambda) d t\right) d x \\
& =\int_{0}^{\infty}\left[g(x)+\int_{x}^{\infty} H(t, x) g(t) d t\right] v_{i}(x, \lambda) d x .
\end{aligned}
$$

The assumption that $\int_{\mathbf{R}} G(\lambda) d\left(\rho_{i j}^{2}\right) G^{*}(\lambda)=0$ implies, using Parseval's equality,

$$
\int_{0}^{\infty}\left[g(x)+\int_{x}^{\infty} H(t, x) g(t) d t\right]^{2} d x=0 .
$$

Now suppose the support of $g$ is contained in the interval $[0, m]$. For almost every $x$,

$$
g(x)+\int_{x}^{\infty} H(t, x) g(t) d t=g(x)+\int_{x}^{m} H(t, x) g(t) d t=0
$$

is a Volterra integral equation with only the trivial solution $g(x) \equiv 0$.

12. Definition. Suppose we are given the two $2 n$th order symmetric differential operators $L^{1}\left(l^{1}, A\right)$ and $L^{2}\left(l^{2}, B\right)$ such that for some kernel $K(x, y)$ we have $\mathcal{K} L^{1}=L^{2} \mathcal{K}$. In Lemma 11 there corresponds to $K(x, y)$ a unique function $F(x, y) \in C^{2 n}$ for $x, y>0$ with various stated properties. We shall say $L^{1}$ is related to $L^{2}$ if (i) $\mathscr{K} L^{1}=L^{2} \mathscr{K}$, (ii) $l_{y}^{1} K=l_{x}^{2} K$, (iii) $A \cdot \hat{F}=(0)$ and (iv) $l_{y}^{1} F=l_{x}^{1} F$.

13. RemarK. No attempt will, or need be, made to establish symmetry or transitivity for this relation. It is designed specifically and solely to establish necessary and sufficient conditions for the solvability of the local inverse spectral problem.

14. Remark. If $2 n=2$ condition (i) in Definition 12 implies the others [3]. 
The next lemma and its proof may be found in the paper of Levitan and Gasymov [3].

15. LemMa. Suppose we are given the integral equation

$$
g(x, y)=h(x, y)+\int_{0}^{1} k(x, y, s) h(x, s) d s
$$

in which the kernel $k(x, y, s)$ and the inhomogeneous term $g(x, y)$ are continuous functions of the parameter $x$ and the independent variable $y$. If, for $x=x_{0}$, the homogeneous equation has only the trivial solution, then in some neighborhood of $x=x_{0}$ the solution $h(x, y)$ is a continuous function of $y$ and $x$. If $k$ and $g$ have $m$ continuous derivatives with respect to $x$ then $h(x, y)$ has too.

16. LEMMA. Given the symmetric differential operator $L^{1}$, a vector of generalized eigenfunctions $u(x, \lambda)$, a determining matrix $\left(\rho_{i j}^{1}\right)$, and some positive matrix measure $\left(\rho_{i j}\right)$ which is adequate with respect to $u(x, \lambda)$ and such that the function

$$
f(x, y) \stackrel{\Delta}{=} \int_{\mathbf{R}} \varphi(x, \lambda) d\left(\rho_{i j}-\rho_{i j}^{1}\right) \varphi^{*}(y, \lambda)
$$

exists and has a mixed derivative

$$
F(x, y) \stackrel{\Delta}{=} \frac{\partial^{2} f}{\partial x \partial y} \in C_{[0, \infty) \times[0, \infty)}^{2 n}
$$

then the integral equation

(1) $F(x, y)+K(x, y)+\int_{0}^{x} K(x, s) F(s, y) d s=0, \quad 0 \leq y \leq x$,

has a unique solution $K(x, y) \in C^{2 n}$ for $0 \leq y \leq x$.

Proof. Arbitrarily fix $x$. Suppose

$$
g(t)+\int_{0}^{x} F(s, t) g(s) d s=0 \quad \text { for } g \in C^{1} .
$$

Multiply and integrate to get

$$
0=\int_{0}^{x}|g(t)|^{2} d t+\int_{0}^{x} \int_{0}^{x} g(s) \frac{\partial^{2} f}{\partial s \partial t} \overline{g(t)} d s d t .
$$


Using $f(s, t)=\overline{f(t, s)}, f(s, 0)=f(0, t)=0$ and integrating by parts we get

(2)

$$
\begin{aligned}
0= & \int_{0}^{x}|g(t)|^{2} d t+|g(x)|^{2} f(x, x)-2 \operatorname{Re}\left[g(x) \int_{0}^{x} f(x, t) \overline{g^{\prime}(t)} d t\right] \\
& +\int_{0}^{x} \int_{0}^{x} g^{\prime}(s) f(s, t) \overline{g^{\prime}(t)} d t d s .
\end{aligned}
$$

Using Parseval's equality we can establish that

$$
\begin{aligned}
\int_{0}^{x}|g(t)|^{2} d t= & \int_{\mathbf{R}_{i, j}}\left(\int_{0}^{x} g(s) u_{i}(s, \lambda) d s\right) d \rho_{l j}(\lambda) \\
& \times\left(\int_{0}^{x} g(t) u_{j}(t, \lambda) d t\right) \\
& -|g(x)|^{2} f(x, x)+2 \operatorname{Re}\left[g(x) \int_{0}^{x} f(x, s) \overline{g^{\prime}(s)} d s\right] \\
& -\int_{0}^{x} \int_{0}^{x} g^{\prime}(s) f(s, t) \overline{g^{\prime}(t)} d s d t .
\end{aligned}
$$

Combining this with (2) we find

$$
0=\int_{\mathbf{R}_{i, j}} \int_{0}^{x} g(s) u_{i}(s, \lambda) d s d \rho_{\imath j}(\lambda) \int_{0}^{x} g(t) u_{j}(t, \lambda) d t,
$$

and, by hypothesis, this implies $\chi_{[0, x]}(t) \cdot g(t) \equiv 0$ so $g(t) \equiv 0$. Now applying the Fredholm alternative to the non-homogeneous equation

$$
F(x, y)+K(x, y)+\int_{0}^{x} K(x, s) F(s, y) d s=0
$$

for fixed $x$, we get the existence and uniqueness of $K(x, y)$ for each $x$.

Finally we rewrite the (1) in order to apply Lemma 15 as follows: replace $y$ by $x y$ and $s$ by $s x$ to get

$$
F(x, x y)+K(x, x y)+\int_{0}^{1} K(x, s x) x F(x y, s x) d s=0 .
$$

Now the kernel $x F(x y, s x)$ and the inhomogeneous term $F(x, x y)$ are, by hypothesis, in $C^{2 n}$ and we've shown the homogeneous equation to have only the trivial solution for any $x$. Lemma 15 implies that, as a function of $x, K$ has $2 n$ continuous derivatives. It is clear that as a function of $y, K$ has as many derivatives as $F(x, y)$.

17. Lemma. Given the two symmetric differential operators $L^{1}$ and $L^{2}$ such that $\mathscr{K} L^{1}=L^{2 \mathscr{K}}$, let $M_{1}=M_{1}\left(p_{1}, \ldots, p_{n}\right)$ and $M_{2}=M_{2}\left(q_{1}, \ldots, q_{n}\right)$ be the $2 n \times 2 n$ matrices that transform by left multiplication column 
vectors $\left(w(0), w^{(1)}(0), \ldots, w^{(2 n-1)}(0)\right)^{\mathrm{T}}$ into the column vectors $\left(w(0), w^{[1]}(0), \ldots, w^{[2 n-1]}(0)\right)^{\mathrm{T}}$ involving quasi-derivatives for $l^{1}$ and $l^{2}$ respectively. Let $T^{-1}=\left(t_{i j}\right)$ be the lower triangular matrix

$$
t_{i j}= \begin{cases}\sum_{k=0}^{i-j-1}\left(\begin{array}{c}
i-2-k \\
j-1
\end{array}\right) K_{k, 0}^{(i-j-k-1)}(0,0) & \text { if } i>j, \\
1, & i=j, \\
0, & i<j .\end{cases}
$$

Then the matrix $A M_{1} T M_{2}^{-1}$ represents the boundary conditions for $L^{2}$. Consequently we may take $B=A M_{1} T M_{2}^{-1}$.

Proof. Recall that if we define $p_{0} \equiv 1$ then $u^{[j]}=u^{(j)}, 0 \leq j \leq n$, and

$$
u^{[n+j]}=\sum_{k=0}^{j}(-1)^{k}\left(p_{j-1} u^{(n-j+1)}\right)^{(k)}, \quad 1 \leq j \leq n,
$$

gives the relationship between regular derivatives and quasi-derivatives for $L^{1}$. So $M_{1}$ is a lower triangular matrix with \pm 1 as diagonal entries:

$$
\left(\begin{array}{c}
u(0) \\
u^{[1]}(0) \\
\vdots \\
u_{x}^{[2 n-1]}(0)
\end{array}\right)=M_{1}\left(\begin{array}{c}
u(0) \\
u^{(1)}(0) \\
\vdots \\
u^{(2 n-1)}(0)
\end{array}\right)
$$

Now

$$
v(x, \lambda)=u(x, \lambda)+\int_{0}^{x} K(x, y) v(y, \lambda) d y
$$

and we check that

$$
\begin{aligned}
& v^{(i-1)}(0, \lambda) \\
& \quad=u_{(0, \lambda)}^{(i-1)}+\sum_{j=1}^{i-1} u^{(j-1)}(0, \lambda)\left(\sum_{l=0}^{i-j-1}\left(\begin{array}{c}
i-2-1 \\
j-1
\end{array}\right) K_{l, 0}^{(i-j-l-1)}(0,0)\right) .
\end{aligned}
$$

From this it is easily demonstrated that

$$
\left(\begin{array}{c}
v(0) \\
v^{(1)}(0) \\
\vdots \\
v^{(2 n-1)}(0)
\end{array}\right)=T^{-1}\left(\begin{array}{c}
u(0) \\
u^{(1)}(0) \\
\vdots \\
u^{(2 n-1)}(0)
\end{array}\right)
$$


where $\lambda$ is suppressed. Furthermore $M_{1}, M_{2}$ and $T$ are clearly invertible so we write

$$
\left(\begin{array}{c}
u(0) \\
u^{[1]}(0) \\
\vdots \\
u^{[2 n-1]}(0)
\end{array}\right)=M_{1} T M_{2}^{-1}\left(\begin{array}{c}
v(0) \\
v^{[1]}(0) \\
\vdots \\
v^{[2 n-1]}(0)
\end{array}\right)
$$

or

$$
\hat{u}(0, \lambda)=M_{1} T M_{2}^{-1} \hat{v}(0, \lambda) .
$$

Now since $A \hat{u}(0, \lambda)=(0)$ this implies

$$
A \cdot M_{1} T M_{2}^{-1} \hat{v}(0, \lambda)=(0) .
$$

Therefore $A M_{1} T M_{2}^{-1}$ must represent the boundary conditions appropriate to $L^{2}$.

18. REMARK. Since $M_{1} T M_{2}^{-1}$ is a lower triangular matrix, then using Lemma 17 non-relatedness (see Definition 12) of certain differential operators because of their boundary conditions becomes apparent. For example, the last column of the boundary matrices must be matchable. That is, there must exist an invertible $n \times n$ matrix $N$ such that the last columns of $N \cdot A$ and $B$ exactly coincide, since all boundary matrices equivalent to $A$ have the form $N \cdot A$. In particular in the second order case (10) will only "match" with another $(10)$. Thus the boundary conditions force the special handling which is given this exceptional case in the literature.

Furthermore, depending on $A$, certain relations may be forced upon $K$ at $(0,0)$ in order that the matrix $T$ produce boundary conditions $A M_{1} T M_{2}^{-1}$ which are selfadjoint.

Of course there are many other more profound obstacles to relatedness.

19. Lemma. Suppose $p_{n-k}(x) \in C^{k}[0, \infty)$ for $k=0, \ldots, n-1$. The equality

$$
\begin{aligned}
& (-1)^{n} u^{(2 n)}+\tilde{p}_{2 n-2}(x) u^{(2 n-2)}+\cdots+\tilde{p}_{0} u \\
& =(-1)^{n} u^{(2 n)}+\sum_{k=0}^{n-1}(-1)^{k}\left(p_{n-k} u^{(k)}\right)^{(k)} \quad \text { for } u \in C_{[0, \infty)}^{2 n}
\end{aligned}
$$


holds if and only if

$$
\tilde{p}_{r}=\sum_{i=[(r+1) / 2]}^{\min (r, n-1)}(-1)^{i}\left(\begin{array}{c}
i \\
r-i
\end{array}\right) p_{n-i}^{(2 l-r)}, \quad r=0,1, \ldots, 2 n-2 .
$$

Proof.

$$
\begin{aligned}
\sum_{m=0}^{2 n-2} \tilde{p}_{m} u^{(m)} & =\sum_{k=0}^{n-1}(-1)^{k}\left(p_{n-k} u^{(k)}\right)^{(k)} \\
& =\sum_{m=0}^{2 n-2} u^{(m)} \sum_{k=[(m+1) / 2]}^{\min (m, n-1)}(-1)^{k}\left(\begin{array}{c}
k \\
m-k
\end{array}\right) p_{n-k}^{(2 k-m)}
\end{aligned}
$$

20. Lemma. Given two symmetric differential operators $L^{1}$ and $L^{2}$ such that $L^{1}$ is related to $L^{2}$, then $\alpha_{r}(x)=0$ for $0 \leq r \leq 2 n-2$.

Proof. Since $A \cdot \hat{F}(x, 0)=(0)$ and since from Lemma 11 we have

$$
F(x, y)+K(x, y)+\int_{0}^{x} K(x, s) F(s, y) d s=0 \text { for } 0 \leq y \leq x,
$$

this implies $A \cdot \hat{K}(x, 0)=(0)$. Here let $u(x, \lambda)$ be any one of the $n$ linearly independent generalized eigenfunctions for $L^{1}$. We have $\mathscr{K} L^{1}=L^{2} \mathcal{K}$, $l_{x}^{2} K=l_{y}^{1} K$, and we recall from $\S 1$ that, since $K$ as a function of $y$ and $u$ as a function of $x$ both satisfy the boundary conditions for $L^{1}$, we must have $\langle K(x, 0), u(0, \lambda)\rangle_{1}=0$.

Using Lemma 19 we can establish that

$$
\begin{aligned}
0= & \left(L^{2}-\lambda\right)\left[u(x, \lambda)+\int_{0}^{x} K(x, t) u(t, \lambda) d t\right]=\left(L^{2}-L^{1}\right)[u(x, \lambda)] \\
& +L^{2}\left[\int_{0}^{x} K(x, t) u(t, \lambda) d t\right]-\int_{0}^{x} K(x, t) L^{1}[u](t, \lambda) d t \\
= & \sum_{r=0}^{2 n-2}\left(\tilde{q}_{r}(x)-\tilde{p}_{r}(x)\right) u^{(r)}(x, \lambda) \\
& +\sum_{k=0}^{2 n-1} \sum_{i=k+1}^{2 n} \tilde{q}_{l}(x) u^{(k)}(x, \lambda) \sum_{j=0}^{i-1-k}\left(\begin{array}{c}
i-1-j \\
k
\end{array}\right) K_{j, 0}^{(l-1-j-k)}(x, x) \\
& +\langle K(x, x), u(x, \lambda)\rangle_{1}-\langle K(x, 0), u(0, \lambda)\rangle_{1} \\
= & \sum_{r=0}^{2 n-2} \alpha_{r}(x) u^{(r)}(x, \lambda) .
\end{aligned}
$$


Now the resulting homogeneous differential equation

$$
\sum_{r=0}^{2 n-2} \alpha_{r}(x) u^{(r)}(x, \lambda)=0
$$

has $n$ linearly independent solutions $u_{1}, u_{2}, \ldots, u_{n}$ all satisfying the $n$ linearly independent boundary conditions represented by the matrix $A$. But this ordinary differential equation has order at most $2 n-2$. Consequently all the coefficients must vanish and we have $\alpha_{r}(x)=0,0 \leq r \leq$ $2 n-2$.

\section{CHAPTER 2}

\section{The Local Inverse Spectral Problem}

21. Given a $2 n$th order symmetric differential operator $L^{1}\left(l^{1}, A\right)$ with $u(x, \lambda)$ a corresponding row vector of $n$ linearly independent generalized eigenfunctions, a determining matrix $\left(\rho_{i j}^{1}\right)$ for $L^{1}$ corresponding to $u(x, \lambda)$, and some $n \times n$ positive matrix measure $\left(\rho_{\imath j}\right)$ which is adequate with respect to $u(x, \lambda)$, suppose

$$
f(x, y)=\int_{\mathbf{R}} \varphi(x, \lambda) d\left(\rho_{\iota_{J}}-\rho_{l j}^{1}\right) \varphi^{*}(y, \lambda)
$$

and

$$
F(x, y)=\partial^{2} f / \partial x \partial y
$$

exist with $F \in C^{2 n}[0, \infty) \times[0, \infty)$. By Lemma 16 there exists a unique solution $K(x, y) \in C^{2 n}$, for $0 \leq y \leq x$, to

(*) $\quad F(x, y)+K(x, y)+\int_{0}^{x} K(x, s) F(s, y) d s=0, \quad 0 \leq y \leq x$.

Then as stated in Remark 5, by knowing $K(x, y)$ the even-indexed conditions $\alpha_{r}(x)=0, r=2 k, k=0,1, \ldots, n-1$, can serve to define $q_{1}, \ldots, q_{n}$. At this point $K$ may or may not allow the odd-indexed conditions $\alpha_{r}(x)=0, r=2 k+1, k=0,1, \ldots, n-2$, to hold. Furthermore $K$ may or may not allow the selfadjointness of the boundary matrix $A M_{1} T M_{2}^{-1}$.

22. Definition. Given $L^{1}\left(l^{1}, A\right), u(x, \lambda)$ and $\left(\rho_{l j}(\lambda)\right)$ as above, let $\left(\rho_{l \jmath}^{1}\right)$ be a determining matrix for $L^{1}$ corresponding to $u(x, \lambda)$. We will say 
$\left(\rho_{l j}^{1}\right)$ is close to $\left(\rho_{l \jmath}\right)$ if:

(i) $\left(\rho_{l j}\right)$ is adequate with respect to $u(x, \lambda)$;

(ii)

$$
f(x, y) \stackrel{\Delta}{=} \int_{\mathbf{R}} \varphi(x, \lambda) d\left(\rho_{i j}-\rho_{i j}^{1}\right) \varphi^{*}(y, \lambda)
$$

and $F(x, y) \stackrel{\Delta}{=} \partial^{2} f / \partial x \partial y$ exist;

(iii) $F \in C^{2 n}[0, \infty) \times[0, \infty)$;

(iv) $A \cdot \hat{F}=(0)$;

(v) $l_{x}^{1} F=l_{y}^{1} F$;

(vi) $\alpha_{r}(x)=0$ for $r=0,1, \ldots, 2 n-2$; and

(vii)

$$
\sum_{k=1}^{n} b_{i k} b_{J, 2 n+1-k}=\sum_{k=1}^{n} b_{i, n+k} b_{j, n+1-k}
$$

where $\left(b_{i_{J}}\right)=A M_{1} T M_{2}^{-1}$.

23. Remark. Definition 22 seems to depend on the choice of determining matrix for $L^{1}$ corresponding to $u(x, \lambda)$. It will be shown in Corollary 26 that this is not the case.

24. Lemma. Given two symmetric differential operators $L^{1}$ and $L^{2}$ such that $\mathcal{K} L^{1}=L^{2} \mathcal{K}$, let $K(x, y)$ be the kernel of the operator $\mathcal{K}, H(x, y)$ the kernel of the inverse operator and $F(x, y)$ the function in Lemma 11. Then

$$
H(x, y)=F(x, y)+\int_{0}^{y} K(y, s) F(x, s) d s .
$$

Proof. Let $u(x, \lambda)$ be a $1 \times n$ vector of generalized eigenfunctions of $L^{1}$ and let $\left(\rho_{l j}^{2}\right)$ be a spectral matrix of some selfadjoint extension of $L^{2}$ corresponding to $v(x, \lambda)=\mathscr{K}[u(x, \lambda)]$.

Fix $x$ arbitrarily and let $h(s)=H(x, s)$. We know $h(s)$ may be expanded as

$$
h(s)=\int_{\mathbf{R}} H(\lambda) d\left(\rho_{t_{J}}^{2}(\lambda)\right) v^{*}(s, \lambda),
$$

where

$$
H(\lambda)=\int_{0}^{\infty} h(s) v(s, \lambda) d s
$$


since $h(s)$ has compact support: $h(s) \stackrel{\Delta}{\equiv} 0$ if $s>x$. For any kernel $J(t, s)$ $\in C^{2 n}$ such that

$$
\int_{0}^{t} v^{*}(s, \lambda) J(t, s) d s<\infty \quad \text { for all } t>0
$$

we can write

$$
\begin{aligned}
\int_{0}^{t} h(s) J(t, s) d s & =\int_{0}^{t}\left(\int_{\mathbf{R}} H(\lambda) d\left(\rho_{i j}^{2}(\lambda)\right) v^{*}(s, \lambda)\right) J(t, s) d s \\
& =\int_{\mathbf{R}} H(\lambda) d\left(\rho_{i j}^{2}(\lambda)\right)\left(\int_{0}^{t} v^{*}(s, \lambda) J(t, s) d s\right) .
\end{aligned}
$$

Now the kernels $H(x, s)$ and $K(x, s)$ determine mappings between $u(x, \lambda)$ and $v(x, \lambda)$ such that

$$
\begin{aligned}
v^{*}(x, \lambda)= & u^{*}(x, \lambda)+\int_{0}^{x} K(x, s) u^{*}(s, \lambda) d s \\
= & v^{*}(x, \lambda)+\int_{0}^{x} H(x, s) v^{*}(s, \lambda) d s \\
& +\int_{0}^{x} K(x, s)\left[v^{*}(s, \lambda)+\int_{0}^{s} H(s, t) v^{*}(t, \lambda) d t\right] d s,
\end{aligned}
$$

so

$$
\begin{aligned}
0= & \int_{0}^{x} H(x, s) v^{*}(s, \lambda) d s+\int_{0}^{x} K(x, s) v^{*}(s, \lambda) d s \\
& +\int_{0}^{x} v^{*}(s, \lambda) \int_{s}^{x} K(x, t) H(t, s) d t d s \\
= & \int_{0}^{t} v^{*}(s, \lambda)\left[H(t, s)+K(t, s)+\int_{s}^{t} K(t, r) H(r, s) d r\right] d s .
\end{aligned}
$$

Letting

$$
J(t, s)=H(t, s)+K(t, s)+\int_{s}^{t} K(t, r) H(r, s) d r
$$

in (1), we find $\int_{0}^{t} v^{*}(s, \lambda) J(t, s) d s=0$ implies

$$
\begin{aligned}
0= & \int_{0}^{t} H(x, s)\left[H(t, s)+K(t, s)+\int_{s}^{t} K(t, r) H(r, s) d r\right] d s \\
= & \int_{0}^{t} H(x, s) H(t, s) d s+\int_{0}^{t} K(t, r) H(x, r) d r \\
& +\int_{0}^{t} \int_{s}^{t} K(t, r) H(x, s) H(r, s) d r d s .
\end{aligned}
$$


Now adding $H(x, t)$ to both sides and interchanging the orders of integration we get

$$
\begin{aligned}
H(x, t)= & {\left[H(x, t)+\int_{0}^{t} H(x, s) H(t, s) d s\right] } \\
& +\int_{0}^{t} K(t, r)\left[H(x, r)+\int_{0}^{r} H(x, s) H(r, s) d s\right] d r .
\end{aligned}
$$

Finally we use Lemma 11 to rewrite the expressions within the brackets to get

$$
H(x, t)=F(x, t)+\int_{0}^{t} K(t, r) F(x, r) d r .
$$

Now we are ready to state and prove the main theorem, which asserts the equivalence of the notions of closeness and relatedness as defined herein.

25. THEOREM. Given two 2 nth order symmetric differential operators $L^{1}$ and $L^{2}$ such that $L^{1}$ is related to $L^{2}$, let the vector $u(x, \lambda)$ represent any $n$ linearly independent generalized eigenfunctions for $L^{1}$ and let $\left(\rho_{i j}^{1}\right)$ be a determining matrix for $L^{1}$ corresponding to $u(x, \lambda)$. Let $\left(\rho_{i j}^{2}\right)$ be a determining matrix for $L^{2}$ corresponding to $v(x, \lambda)=\mathcal{K}[u(x, \lambda)]$. Then $\left(\rho_{l j}^{1}\right)$ is close to $\left(\rho_{i j}^{2}\right)$.

Conversely, given a symmetric differential operator $L^{1}$, a vector of generalized eigenfunctions $u(x, \lambda)$ for $L^{1}$, a determining matrix $\left(\rho_{i j}^{1}\right)$ for $L^{1}$ corresponding to $u(x, \lambda)$ and some positive matrix measure $\left(\rho_{i j}\right)$ such that $\left(\rho_{i j}^{1}\right)$ is close to $\left(\rho_{i j}\right)$ then there exists a symmetric differential operator $L^{2}$ for which $\left(\rho_{i j}\right)$ is a determining matrix such that $L^{1}$ is related to $L^{2}$.

Proof. (related $\Rightarrow$ close) Given $L^{1}\left(l^{1}, A\right)$ and $L^{2}\left(l^{2}, B\right)$ suppose $L^{1}$ is related to $L^{2}$. Let $u(x, \lambda),\left(\rho_{i j}^{1}\right)$ and $\left(\rho_{i j}^{2}\right)$ be as hypothesized and let $\left(\sigma_{i j}\right)=\left(\rho_{i j}^{2}-\rho_{i j}^{1}\right)$. By Lemma $11\left(\rho_{i j}^{2}\right)$ is adequate with respect to $u(x, \lambda)$,

$$
f(x, y)=\int_{\mathbf{R}}\left[\int_{0}^{x} u(x, \lambda) d s\right] d\left(\sigma_{i j}(\lambda)\right)\left[\int_{0}^{y} u(t, \lambda) d t\right]^{*}
$$

exists and has a mixed partial derivative

$$
F(x, y)=\partial^{2} f / \partial x \partial y \in C_{[0, \infty) \times[0, \infty)}^{2 n} .
$$

From Definition $12 A \cdot \hat{F}=(0)$ and $l_{x}^{1} F=l_{y}^{1} F$. By Lemma $20 \alpha_{r}(x)=0$ for $r=0,1, \ldots, 2 n-2$. By Lemma 17 the matrix $A M_{1} T M_{2}^{-1}$ is equivalent 
to the selfadjoint boundary matrix $B$ for $L^{2}$ and is therefore also selfadjoint.

(close $\Rightarrow$ related) Given $L^{1}\left(l^{1}, A\right), u(x, \lambda),\left(\rho_{i j}^{1}\right)$ and some positive matrix measure $\left(\rho_{i j}\right)$ such that $\left(\rho_{i j}^{1}\right)$ is close to $\left(\rho_{i j}\right)$, let $\left(\sigma_{i j}\right)=\left(\rho_{i j}-\rho_{i j}^{1}\right)$. From Definition $6\left(\rho_{i j}\right)$ is adequate with respect to $u(x, \lambda),(\dagger)$ and $F(x, y)=\partial^{2} f / \partial x \partial y$ exist, and $F \in C_{[0, \infty) \times[0, \infty)}^{2 n}$ By Lemma $16(*)$ has a unique solution $K(x, y) \in C^{2 n}$ for $0 \leq y \leq x$.

Let

$$
I(x, y)=F(x, y)+K(x, y)+\int_{0}^{x} K(x, s) F(s, y) d s .
$$

The identity $I \equiv 0$ clearly implies

$$
A \cdot \hat{K}(x, 0)=A \cdot \hat{F}(x, 0)=0=A \cdot \hat{F}(0, y) .
$$

Hence $\langle K(x, 0), F(0, y)\rangle_{1}=0$. Now

$$
\begin{aligned}
&\left.\langle K(x, t), F(t, y)\rangle_{1}\right|_{t=x} \sum_{m=1}^{n}(-1)^{m}\left(\sum_{i=0}^{m-1}(-1)^{i} F_{i, 0}(x, y) \frac{\partial^{m-1-i}}{\partial t^{m-1-i}}\left[p_{n-m}(t) K_{0, m}(x, t)\right]_{t=x}\right. \\
&\left.\quad-\sum_{i=0}^{m-1}(-1)^{i} K_{0, i}(x, x) \frac{\partial^{m-1-i}}{\partial t^{m-1-i}}\left[p_{n-m}(t) F_{m, 0}(t, y)\right]_{t=x}\right) \\
&= \sum_{m=1}^{n} \sum_{i=0}^{m-1}(-1)^{m+i} F_{i, 0}(x, y) \frac{\partial^{m-1-i}}{\partial t^{m-1-i}}\left[p_{n-m}(t) K_{0, m}(x, t)\right]_{t=x} \\
&-\sum_{m=1}^{n} \sum_{i=0}^{m-1} \sum_{r=0}^{m-1-i}(-1)^{m+i} K_{0, i}(x, x)(m-1-i) \\
&= \sum_{r=0}^{n-1} F_{r, 0}(x, y) \sum_{m=r+1}^{n}(-1)^{m+r} \frac{\partial^{m-1-r}}{\partial t^{m-1-r}}\left[p_{n-m}(r) K_{0, m}^{(m-1-i-r)}(x) F_{m+r, 0}(x, y)\right]_{t=x} \\
&-\sum_{r=1}^{2 n-1} F_{r, 0}(x, y) \sum_{m=[(r+2) / 2]}^{\min (r, n)} \sum_{i=0}^{2 m-1-r}(-1)^{m+i} K_{0, i}(x, x)(m-1-i) \\
& r-m
\end{aligned}
$$

where the middle index $m$ in this last expression may start at 1 since the innermost summation forces $2 m-1-r \geq 0$, which implies $m \geq$ $(r+1) / 2$, and since $m$ is an inter,

$$
m \geq\left[\frac{r+2}{2}\right] \geq \frac{r+1}{2}>\left[\frac{r+2}{2}\right]-1
$$

is automatic. 
Now using the hypothesis $l_{x}^{1}(F(x, y))=l_{y}^{1}(F(x, y))$ and using the expanded form for the differential expression $l^{1}$ involving the coefficients $\tilde{q}_{l}, i=0,1, \ldots, 2 n$, we consider the identity

$$
\begin{aligned}
0 \equiv & \left(l_{x}^{2}-l_{y}^{1}\right) I \\
= & \left(l_{x}^{2}-l_{y}^{1}\right) K(x, y)+\int_{0}^{x}\left(l_{x}^{2}-l_{s}^{1}\right)[K(x, s)] F(s, y) d s \\
& +\sum_{k=0}^{2 n-2} F_{k, 0}(x, y) \alpha_{k}(x) .
\end{aligned}
$$

By hypothesis $\alpha_{k}(x)=0$ for $k=0,1, \ldots, 2 n-2$, so letting $g(x, y)=$ $\left(l_{x}^{2}-l_{y}^{1}\right) K(x, y)$, we have the homogeneous equation

$$
g(x, y)=\int_{0}^{x} g(x, s) F(s, y) d s=0,
$$

which, for each fixed $x$, has been shown to have only the trivial solution in the proof of Lemma 16. Hence $l_{x}^{2}(K(x, y))=l_{y}^{1}(K(x, y))$. This will now be shown to imply $v(x, \lambda)=\mathscr{K}[u(x, \lambda)]$ satisfies $l^{2} v=\lambda v$ whenever $l^{1} u=\lambda u$ and $A \cdot u(0, \lambda)=(0)$.

Recalling that $\tilde{q}_{2 n}(x) \equiv(-1)^{n}, \tilde{q}_{2 n-1}(x) \equiv 0$, we have

$$
\begin{aligned}
l^{2}[v(x, \lambda)]= & l^{2}[u(x, \lambda)]+\int_{0}^{x} l_{x}^{2}[K(x, y)] u(y, \lambda) d y \\
& +\sum_{m=1}^{2 n} \tilde{q}_{m}(x) \sum_{r=0}^{m-1}\left[u(x, \lambda) K_{r, 0}(x, x)\right]^{(m-1-r)} .
\end{aligned}
$$

On the other hand, since $A \cdot \hat{K}(x, 0)=(0)$ implies

$$
\left.\langle K(x, y), u(y, \lambda)\rangle_{1}\right|_{y=0}=0
$$

we have

$$
\begin{aligned}
\lambda v(x, \lambda)=\lambda u(x, \lambda)+\int_{0}^{x} K(x, y) \lambda u(y, \lambda) d y \\
=l^{1}(u(x, \lambda))+\int_{0}^{x} K(x, y) l^{1}[u(y, \lambda)] d y \\
=l^{1}[u(x, \lambda)]+\int_{0}^{x} l_{y}^{1}[K(x, y)] u(y, \lambda) d y \\
\\
-\langle K(x, y), u(y, \lambda)\rangle_{1} l_{y=x} .
\end{aligned}
$$

Combining (2) and (3) we can establish

$$
\left(l^{2}-\lambda\right) v(x, \lambda)=\sum_{r=0}^{2 n-2} u^{(r)}(x, \lambda) \alpha_{r}(x) \equiv 0 .
$$


Now as in Lemma 17 we let $M_{1}$ and $M_{2}$ be the matrices which, by left multiplication, transform column vectors

$$
\left(w(0), w^{(1)}(0), \ldots, w^{(2 n-1)}(0)\right)^{\mathrm{T}}
$$

into column vectors

$$
\left(w(0), w^{[1]}(0), \ldots, w^{[2 n-1]}(0)\right)^{\mathrm{T}}
$$

involving quasi-derivatives for $l^{1}$ and $l^{2}$ respectively. Define the lower triangular matrix $T^{-1}=\left(t_{i j}\right)$, where

$$
t_{t j}= \begin{cases}\sum_{k=0}^{i-j-1}\left(\begin{array}{c}
i-2-k \\
j-1
\end{array}\right) K_{k, 0}^{(l-\jmath-k-1)}(0,0), & i>j, \\
1, & i=j, \\
0, & i<j .\end{cases}
$$

Now $v(x, \lambda)=u(x, \lambda)+\int_{0}^{x} K(x, y) u(y, \lambda) d y$ implies

$$
\begin{aligned}
v^{(i-1)}(0, \lambda)= & u^{(t-1)}(0, \lambda) \\
& +\sum_{j=1}^{i-1} u^{(j-1)}(0, \lambda)\left(\sum_{k=0}^{i-j-1}\left(\begin{array}{c}
i-2-k \\
j-1
\end{array}\right) K_{k, 0}^{(i-j-k-1)}(0,0)\right),
\end{aligned}
$$

or, in other words,

$$
\left(\begin{array}{c}
v(0, \lambda) \\
v^{(1)}(0, \lambda) \\
\vdots \\
v^{(2 n-1)}(0, \lambda)
\end{array}\right)=T^{-1}\left(\begin{array}{c}
u(0, \lambda) \\
u^{(1)}(0, \lambda) \\
\vdots \\
u^{(2 n-1)}(0, \lambda)
\end{array}\right)
$$

which implies $\hat{u}(0, \lambda)=M_{1} T M_{2}^{-1} \hat{v}(0, \lambda)$. So if we define the matrix $B=A M_{1} T M_{2}^{-1}$ which represents selfadjoint boundary conditions by hypothesis, and use $l^{2}$ and $B$ to define a symmetric differential operator $L^{2}$, then $v(x, \lambda)=\mathscr{K}[u(x, \lambda)]$ clearly satisfies $B \hat{v}(0, \lambda)=0$ whenever $A$. $\hat{u}(0, \lambda)=0$. Also $\hat{v}(0, \lambda)=M_{2} T^{-1} M_{1}^{-1} \hat{u}(0, \lambda)$ is independent of $\lambda$ and therefore $v(x, \lambda)$ represents the generalized eigenfunction for $L^{2}$ whenever $u(x, \lambda)$ represents those for $L^{1}$, that is, $\mathscr{K} L^{1}=L^{2} \mathcal{K}$.

It remains to verify that the given positive matrix measure $\left(\rho_{l j}\right)$ is indeed a determining matrix for $L^{2}$ corresponding to $v(x, \lambda)=\mathscr{K}[u(x, \lambda)]$ by demonstrating the validity of Parseval's equality. 
For $g(x) \in \mathcal{L}^{2}[0, \infty)$ with compact support let

$$
\begin{aligned}
G(\lambda) & =\int_{0}^{\infty} g(t) v(t, \lambda) d t \\
& =\int_{0}^{\infty} g(t)\left[u(t, \lambda)+\int_{0}^{t} K(t, s) u(s, \lambda) d s\right] d t \\
& =\int_{0}^{\infty} g(s) u(s, \lambda) d s+\int_{0}^{\infty} u(s, \lambda)\left[\int_{s}^{\infty} K(t, s) g(t) d t\right] d s \\
& =\int_{0}^{\infty} h(s) u(s, \lambda) d s,
\end{aligned}
$$

where

$$
h(s) \stackrel{\Delta}{=} g(s)+\int_{s}^{\infty} K(t, s) g(t) d t .
$$

Since $h \in \mathfrak{L}^{2}[0, \infty)$ and has compact support then, by a symmetric argument, we also have

$$
\int_{0}^{\infty} h(s) u(s, \lambda) d s=\int_{0}^{\infty} f(t) v(t, \lambda) d t
$$

where

$$
f(t) \stackrel{\Delta}{=} h(t)+\int_{t}^{\infty} H(s, t) h(s) d s
$$

This means

$$
g(t)=h(t)+\int_{t}^{\infty} H(s, t) h(s) d s
$$

since the $v$-transform defines an isometric, hence, injective mapping of $\mathfrak{L}^{2}[0, \infty)$ into $\mathcal{L}^{2}\left(\mathbf{R},\left(\rho_{i j}^{2}\right)\right)$ where $\left(\rho_{i j}^{2}\right)$ is a spectral matrix for $L^{2}\left(l^{2}, B\right)$.

It may be shown that

$$
\int_{\mathbf{R}} G(\lambda) d\left(\rho_{i j}\right) G^{*}(\lambda)=\int_{0}^{\infty} h^{2}(t) d t+\int_{0}^{\infty} \int_{0}^{\infty} h(s) h(t) F(s, t) d s d t .
$$

Recalling from Lemma 24 the identity

$$
H(s, t)=F(s, t)+\int_{0}^{t} K(t, r) F(s, r) d s \quad(0 \leq t \leq s),
$$

and recalling the integral equation

$$
-K(t, s)=F(s, t)+\int_{0}^{t} K(t, r) F(s, r) d r \quad(0 \leq s \leq t),
$$


we can establish that

$$
\int_{0}^{\infty} h(t) F(s, t) d t=\int_{0}^{s} g(t) H(s, t) d t-\int_{s}^{\infty} g(t) K(t, s) d t .
$$

Therefore

$$
\begin{aligned}
\int_{\mathbf{R}} G(\lambda) d\left(\rho_{i j}\right) G^{*}(\lambda)= & \int_{0}^{\infty} h^{2}(t) d t+\int_{0}^{\infty} h(s) \int_{0}^{s} g(t) H(s, t) d t d s \\
& -\int_{0}^{\infty} h(s) \int_{s}^{\infty} g(t) K(t, s) d t d s \\
= & \int_{0}^{\infty} h^{2}(t) d t+\int_{0}^{\infty} g(t)\left[\int_{t}^{\infty} H(s, t) h(s) d s\right] d t \\
& -\int_{0}^{\infty} h(s)\left[\int_{s}^{\infty} g(t) K(t, s) d t\right] d s .
\end{aligned}
$$

Now from the definition (4) of $h$,

$$
\int_{s}^{\infty} g(t) K(t, s) d t=h(s)-g(s)
$$

and from observation (5) we have

$$
\int_{t}^{\infty} h(s) H(s, t) d s=g(t)-h(t) .
$$

Substitution now gives

$$
\begin{aligned}
\int_{\mathbf{R}} G(\lambda) d\left(\rho_{i j}^{2}(\lambda)\right) G^{*}(\lambda)= & \int_{0}^{\infty} h^{2}(t) d t+\int_{0}^{\infty} g(t)[g(t)-h(t)] d t \\
& -\int_{0}^{\infty} h(s)[h(s)-g(s)] d s \\
= & \int_{0}^{\infty} g^{2}(t) d t
\end{aligned}
$$

which proves Parseval's equation. The generalized Parseval equality follows by the polarization identity. Thus the $v$-transform

$$
f(x) \mapsto \int_{0}^{\infty} f(x) v(x, \lambda) d x
$$

defines an isometry $V: \mathfrak{L}^{2}[0, \infty) \rightarrow \mathfrak{L}^{2}\left(\mathbf{R},\left(\rho_{i j}\right)\right)$. For any function $f$ with compact support in the comain $\mathscr{D}^{2}$ of $L^{2}\left(l^{2}, B\right)$ we have

$$
\begin{aligned}
\int_{0}^{\infty} l^{2}[f(x)] v(x, \lambda) d x= & \lim _{x \rightarrow \infty}\langle f(x), v(x, \lambda)\rangle_{2}-\langle f(0), v(0, \lambda)\rangle_{2} \\
& +\int_{0}^{\infty} f(x) l^{2}[v(x, \lambda)] d x \\
= & \lambda \int_{0}^{\infty} f(x) v(x, \lambda) d x .
\end{aligned}
$$


Since functions such as $f$ are dense in $\mathscr{D}^{2}$ and since $V$ is isometric, it follows that $V\left(\mathscr{D}^{2}\right) \subset \mathscr{D}_{\Lambda}$, and for $f \in \mathscr{D}^{2}$ we have $V \circ L^{2}[f]=\Lambda \circ V[f]$. Thus $\left(\rho_{i j}\right)$ is a determining matrix for $L^{2}$ corresponding to $v(x, \lambda)=$ $\mathscr{K}[u(x, \lambda)]$.

26. CoROllary. Let $\left(\rho_{i j}\right)$ and $\left(\tilde{\rho}_{i j}\right)$ be determining matrices for $L^{1}$ corresponding to $u(x, \lambda)$ and let $\left(\rho_{i j}^{2}\right)$ be a positive matrix measure. If $\left(\rho_{i j}\right)$ is close to $\left(\rho_{i j}^{2}\right)$ then $\left(\tilde{\rho}_{i j}\right)$ is close to $\left(\rho_{i j}^{2}\right)$.

Proof. If $\left(\rho_{i j}\right)$ is close to $\left(\rho_{i j}^{2}\right)$ then there exists a symmetric differential operator $L^{2}$ for which $\left(\rho_{i j}^{2}\right)$ is a determining matrix such that $L^{1}$ is related to $L^{2}$. From this it follows that $\left(\tilde{\rho}_{i j}\right)$ is close to $\left(\rho_{i j}^{2}\right)$.

27. Corollary. A determining matrix uniquely determines a symmetric differential operator on $\mathfrak{L}^{2}[0, \infty)$.

Proof. Suppose $\left(\rho_{i j}\right)$ is a determining matrix for $L^{1}$ corresponding to $u(x, \lambda)$ and, at the same time, for $L^{2}$. We will use the local inverse scheme to construct $L^{2}$ from $\left(\rho_{i j}\right)$.

Parseval's equality implies $\left(\rho_{i j}\right)$ is adequate with respect to $u(x, \lambda)$. Certainly $\left(\rho_{i j}\right)$ is close to $\left(\rho_{i j}\right)$ because

$$
f(x, y) \triangleq \int \varphi(x, \lambda) d\left(\rho_{i j}(\lambda)-\rho_{i j}(\lambda)\right) \varphi^{*}(y, \lambda) \equiv 0,
$$

$F(x, y)=\partial^{2} f / \partial x \partial y \equiv 0$ and $K(x, y) \equiv 0$. This defines, by the conditions $\alpha_{r}(x)=0$ and $B=A \cdot M_{1} T M_{2}^{-1}$, a symmetric differential operator $L^{2} \equiv L^{1}$.

28. Summary remarks. We now have the techniques to solve the local inverse spectral problem.

Suppose we are given a symmetric differential operator $L^{1}$ of order $2 n$ on the interval $[0, \infty)$, a vector of $n$ linearly independent generalized eigenfunctions $u(x, \lambda)$ for $L^{1}$, and some $n \times n$ positive matrix measure ( $\left.\rho_{i j}\right)$ such that a determining matrix $\left(\rho_{i j}^{1}\right)$ for $L^{1}$ corresponding to $u(x, \lambda)$ is close to $\left(\rho_{i j}\right)$. Using $u(x, \lambda)$ and $\left(\sigma_{i j}\right)=\left(\rho_{i j}-\rho_{i j}^{1}\right)$ we construct

$$
f(x, y)=\int_{\mathbf{R}} \varphi(x, \lambda) d\left(\sigma_{i j}(\lambda)\right) \varphi^{*}(y, \lambda),
$$

take the mixed partial derivative $F(x, y)=\partial^{2} f / \partial x \partial y$ and set up the integral equation

(ł) $F(x, y)+K(x, y)+\int_{0}^{x} K(x, s) F(s, y) d s=0, \quad 0 \leq y \leq x$. 
Then we find the unique solution $K(x, y)$ and use the conditions $\alpha_{r}(x)=0$ for $r=0,1, \ldots, 2 n-2$ to define the coefficients $q_{1}(x), \ldots, q_{n}(x)$ of a formally selfadjoint differential expression $l^{2}$. Finally we define $M_{2}$ and the boundary conditions $B=A M_{1} T M_{2}^{-1}$ which, together with $l^{2}$, define a symmetric differential operator $L^{2}$ for which the given positive matrix measure is a determining matrix.

\section{APPENDIX}

1. EXAmple. This example illustrates that the conditions given by Sahnovič are in fact not necessary for the solution of the local inverse spectral problem.

Let $L(p, \alpha)$ be the operator with boundary condition $\sin (\alpha) \cdot u(0)+$ $\cos (\alpha) \cdot u^{\prime}(0)=0$, where $L(p, \alpha)[u]=-u^{\prime \prime}+p u$. It is known that $L(0,0)$ is related to $L(0, \alpha)$, where $\alpha \neq \pi / 2(\pi)$. In this case the function $f(x, y)$ of Lemma 11 is differentiable under the integral sign and $F(x, y)=$ $\partial^{2} f / \partial x \partial y$ has the integral representation

$$
\begin{aligned}
F(x, y) & =\int_{0}^{\infty} \cos \sqrt{\lambda} x \cos \sqrt{\lambda} y\left(\frac{\sqrt{\lambda}}{h^{2}+\lambda}-\frac{1}{\sqrt{\lambda}}\right) \frac{d \lambda}{\pi} \\
& =\int_{0}^{\infty} \cos \sqrt{\lambda} x \cos \sqrt{\lambda} y\left(\frac{-h^{2}}{\sqrt{\lambda}\left(h^{2}+\lambda\right)}\right) \frac{d \lambda}{\pi} \quad \text { where } h=\frac{-\sin \alpha}{\cos \alpha} .
\end{aligned}
$$

Here the conditions of Sahnovič would require the two-fold differentiability of $F$ under the integral sign.

Attemping to differentiate twice under the integral with respect to either variable would give an integral expression asymptotic to $\int_{0}^{\infty} \cos \sqrt{\lambda} x \cos \sqrt{\lambda} y d \lambda / \sqrt{\lambda}$, which does not exist. In this case, however, it is known that $K(x, y) \equiv h$ and by solving ( $\ddagger)$ for $F$ we find

$$
F(x, y)=-(h / 2)\left[e^{-h(x+y)}+e^{-h(x-y)}\right] \text { for } y \leq x .
$$

2. EXAMPLE. The class of spectral matrices for a symmetric differential operator is, in general, smaller than the class of its determining matrices.

Let $l$ be a second order Sturm-Liouville differential expression in the limit circle case. Let $\hat{\rho}$ and $\hat{\hat{\rho}}$ be non-equivalent spectral measures for two distinct selfadjoint extensions of $L(l, A)$ corresponding to the generalized eigenfunction $u(x, \lambda)$. The rule

$$
f(x) \mapsto \int_{0}^{\infty} f(x) u(x, \lambda) d x
$$


defines an isometry of $\varrho^{2}[0, \infty)$ onto $\mathcal{L}^{2}(\mathbf{R}, \hat{\rho})$ and an isometry of $\mathcal{L}^{2}[0, \infty)$ onto $\mathfrak{L}^{2}(\mathbf{R}, \hat{\rho})$. If we let $\rho=\frac{1}{2}(\hat{\rho}+\hat{\rho})$ it is easily seen that the above rule also defines an isometry $U$ of $\mathcal{L}^{2}[0, \infty)$ into $\mathcal{L}^{2}(\mathbf{R}, \rho)$.

For the non-equivalent atomic measures $\hat{\rho}$ and $\hat{\hat{\rho}}$ find a point $\lambda_{0} \in \mathbf{R}$ such that $\hat{\hat{\rho}}\left(\left\{\lambda_{0}\right\}\right)>0$ and $\hat{\rho}\left(\left\{\lambda_{0}\right\}\right)=0$. Suppose for some $f \in \mathcal{L}^{2}[0, \infty)$ with

$$
F(\lambda)=\int_{0}^{\infty} f(x) u(x, \lambda) d x
$$

we have

$$
\left\|\chi_{\left\{\lambda_{0}\right\}}(\lambda)-F(\lambda)\right\|_{\rho}^{2}=0
$$

Then we have

$$
0=\frac{1}{2}\left\|\chi_{\left\{\lambda_{0}\right\}}(\lambda)-F(\lambda)\right\|_{\hat{\rho}}^{2}+\frac{1}{2}\left\|\chi_{\left\{\lambda_{0}\right\}}(\lambda)-F(\lambda)\right\|_{\hat{\rho}}^{2},
$$

which implies $F=0 \hat{\rho}$-a.e. This leads to the contradiction

$$
0=\|F\|_{\hat{\rho}}^{2}=\|f\|^{2}=\|F\|_{\hat{\rho}}^{2}=\left\|\chi_{\left\{\lambda_{0}\right\}}(\lambda)\right\|_{\hat{\rho}}^{2}=\hat{\hat{\rho}}\left(\left\{\lambda_{0}\right\}\right)>0 .
$$

Thus $\chi_{\left\{\lambda_{0}\right\}}(\lambda)$ is an element of $\mathcal{L}^{2}(\mathbf{R}, \rho)$ which is not the image under the mapping $U$ of any $f \in \mathcal{L}^{2}[0, \infty)$. Consequently $U$ is not onto and $\rho$ cannot be a spectral measure for $L(l, A)$.

\section{REFERENCES}

[1] N. Dunford and J. T. Schwartz, Linear Operators, Part II, N. Y. Pub. John Wiley \& Sons, 1963.

[2] I. M. Gelfand and B. M. Levitan, On the determination of a differential equation by its spectral function, Amer. Math. Soc., Translations Series 2, 1 (1955), 253-304.

[3] B. M. Levitan and M. G. Gasymov, Determination of a differential equation by two of its spectra, Russian Math. Surveys, 19 (1964), (2), 1-63.

[4] M. A. Naimark, Linear Differential Operators in Hilbert Space, 2nd Ed., Vol. II, New York: Frederick Ungar, 1968.

[5] M. Rosenberg, The square integrability of matrix-valued functions with respect to a non-negative Hermitian measure, Duke Math. J., 31 (1964), 291-298.

[6] L. A. Sahnovič, Transformation operators methods for equations of higher order, Mat. Sb., T55 (97) \#3, (1961), 347-360.

[7] M. H. Stone, Linear Transformations in Hilbert Space, Amer. Math. Soc. Colloq. Publ., Vol. XV, N. Y., 1932.

Received January 27, 1982 and in revised form September 14, 1982. 



\section{PACIFIC JOURNAL OF MATHEMATICS}

EDITORS

Donald BabBITT (Managing Editor)

University of California

Los Angeles, CA 90024

Hugo Rossi

University of Utah

Salt Lake City, UT 84112

C. C. Moore and Arthur Ogus

University of California

Berkeley, CA 94720
J. DugunduI

Department of Mathematics

University of Southern California

Los Angeles, CA 90089-1113

R. FINN and H. SAMELSON

Stanford University

Stanford, CA 94305

\section{ASSOCIATE EDITORS}
R. ARENS
E. F. BECKENBACH
B. H. NeumanN
F. WolF
K. YosHIDA (1906-1982)

\section{SUPPORTING INSTITUTIONS}

UNIVERSITY OF ARIZONA

UNIVERSITY OF BRITISH COLUMBIA

CALIFORNIA INSTITUTE OF TECHNOLOGY

UNIVERSITY OF CALIFORNIA

MONTANA STATE UNIVERSITY

UNIVERSITY OF NEVADA. RENO

NEW MEXICO STATE UNIVERSITY

OREGON STATE UNIVERSITY
UNIVERSITY OF OREGON

UNIVERSITY OF SOUTHERN CALIFORNIA

STANFORD UNIVERSITY

UNIVERSITY OF HAWAII

UNIVERSITY OF TOKYO

UNIVERSITY OF UTAH

WASHINGTON STATE UNIVERSITY

UNIVERSITY OF WASHINGTON 


\section{Pacific Journal of Mathematics}

\section{Vol. 111, No. $1 \quad$ November, 1984}

Harald Brandenburg and Adam Stefan Mysior, For every Hausdorff

space $Y$ there exists a nontrivial Moore space on which all continuous

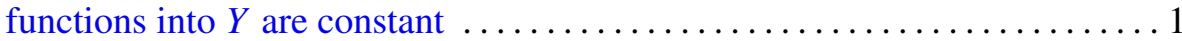

Henry Dappa, A Marcinkiewicz criterion for $L^{p}$-multipliers $\ldots \ldots \ldots \ldots \ldots 9$

P. H. Doyle, III and John Gilbert Hocking, Bijectively related spaces. I.

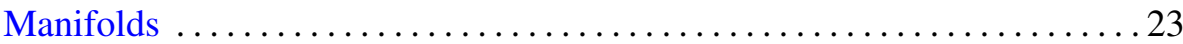

Joel Hass, Complete area minimizing minimal surfaces which are not totally

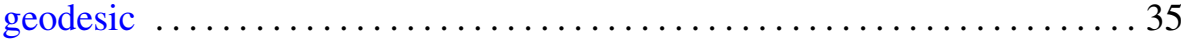

Aarno Hohti, On Ginsburg-Isbell derivatives and ranks of metric spaces .... 39

Richard Howard Hudson, Diophantine determinations of $3^{(p-1) / 8}$ and $5^{(p-1) / 4}$

A. F. Izé and A. Ventura, Asymptotic behavior of a perturbed neutral functional-differential equation related to the solution of the unperturbed

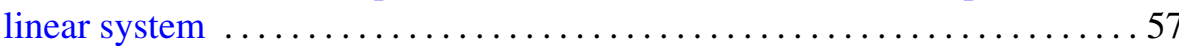

Palle E. T. Jorgensen, Spectral representations of unbounded nonlinear

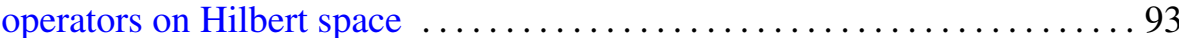

Darrell Conley Kent and Gary Douglas Richardson, Cauchy spaces with

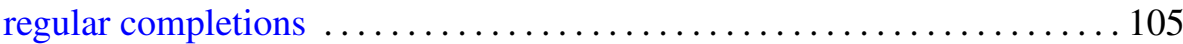

Mark Mahowald, An addendum to: "bo-resolutions" ................ 117

Stuart Wayne Margolis and Jean-Eric Pin, Minimal noncommutative varieties and power varieties

Carla Massaza and Alfio Ragusa, Some conditions on the homology groups of the Koszul complex

Vicente Miquel Molina, Some examples of Riemannian almost-product manifolds

Roderic Murufas, Inverse spectral problems for certain differential operators

Ulrich Oertel, Closed incompressible surfaces in complements of star links

Katsuro Sakai, A characterization of local equiconnectedness

William Victor Smith and Don Harrell Tucker, Weak integral convergence theorems and operator measures 